(2) OPEN ACCESS

\title{
The role of gut microbiota in cancer treatment: friend or foe?
}

\author{
Wing Yin Cheng, ${ }^{1}$ Chun-Ying Wu, ${ }^{2}$ Jun Yu (1) ${ }^{1}$
}

Additional material is published online only. To view please visit the journal online (http://dx.doi.org/10.1136/ gutjnl-2020-321153).

${ }^{1}$ State Key Laboratory of Digestive Disease, Institute of Digestive Disease and The Department of Medicine and Therapeutics, Li Ka Shing Institute of Health Sciences, CUHK Shenzhen Research Institute, The Chinese University of Hong Kong, Hong Kong SAR, China

2Division of Translational Research, Department of Medical Research, Taipei Veterans General Hospital, Taipei, Taiwan; Institute of Biomedical Bioinformatics and School of Medicine, National Yang-Ming University, Taipei, Taiwan; College of Public Health and Graduate Institute of Clinical Medicine, China Medical University, Taichung, Taiwan

\section{Correspondence to}

Dr Jun Yu, Institute of Digestive Disease and The Department of Medicine and Therapeutics, Chinese University of Hong Kong, New Territories, Hong Kong; junyu@cuhk.edu.hk

Received 18 March 2020 Revised 2 July 2020

Accepted 6 July 2020

Published Online First

5 August 2020

\section{ABSTRACT}

The gut microbiota has been implicated in cancer and shown to modulate anticancer drug efficacy. Altered gut microbiota is associated with resistance to chemo drugs or immune checkpoint inhibitors (ICls), whereas supplementation of distinct bacterial species restores responses to the anticancer drugs. Accumulating evidence has revealed the potential of modulating the gut microbiota to enhance the efficacy of anticancer drugs. Regardless of the valuable findings by preclinical models and clinical data of patients with cancer, a more thorough understanding of the interactions of the microbiota with cancer therapy helps researchers identify novel strategy for cancer prevention, stratify patients for more effective treatment and reduce treatment complication. In this review, we discuss the scientific evidence on the role of gut microbiota in cancer treatment, and highlight the latest knowledge and technologies leveraged to target specific bacteria that contribute to tumourigenesis. First, we provide an overview of the role of the gut microbiota in cancer, establishing the links between bacteria, inflammation and cancer treatment. Second, we highlight the mechanisms used by distinct bacterial species to modulate cancer growth, immune responses, as well as the efficacy of chemotherapeutic drugs and ICls. Third, we demonstrate various approaches to modulate the gut microbiota and their potential in translational research. Finally, we discuss the limitations of current microbiome research in the context of cancer treatment, ongoing efforts to overcome these challenges and future perspectives.

\section{INTRODUCTION}

The gut microbiota has emerged as a critical player in the maintenance of human health, influencing not only the GI tract but also distal organs such as the brain, liver and pancreas. ${ }^{12}$ As such, dysbiosis, which refers to compositional and functional alterations of the gut microbiome, contributes to the development of various pathological conditions, including obesity, ${ }^{3-5}$ diabetes, ${ }^{6-8}$ neurodegenerative diseases ${ }^{910}$ and cancers. ${ }^{11-14}$ Notably, bacterial infection can induce cancer. The colonisation of Helicobacter pylori causes persistent inflammation and leads to gastritis, driving gastric malignancy in a portion of infected individuals. Further analysis revealed that $H$.pylori promotes tumourigenesis via the activation of $\beta$-catenin signalling pathway. ${ }^{15}$ Conversely, eradication of H.pylori decreases the risk of gastric cancer in infected individuals, supporting its role in early stages of gastric carcinogenesis. ${ }^{16}$ Likewise, colorectal cancer (CRC), the

\section{Key messages}

- The gut microbiota has been implicated in cancer and shown to modulate the efficacy of anticancer treatment.

- Altered gut microbiota is associated with resistance to chemo drugs or immune checkpoint inhibitors (ICIs). Modulating the microbiota via antibiotics, probiotics, faecal microbiota transplant or nanotechnologies may potentiate the antitumour effects of chemo drugs or ICls.

- Preclinical and clinical studies suggest the use of tumour-associated bacteria as diagnostic or prognostic markers for cancer.

- Microbial signature may be used to stratify patients with cancer for more effective treatment or to reduce treatment complication.

development of cancer from the colon, is associated with specific bacteria. Recently, analysis of faecal metagenomic samples from patients with CRC identified CRC-enriched bacteria, including Bacteroides fragilis, Fusobacterium nucleatum, Porphyromonas asaccharolytica, Parvimonas micra, Prevotella intermedia, Alistipes finegoldii and Thermanaerovibrio acidaminovorans, which may potentially serve as diagnostic bacterial markers across populations. ${ }^{1718}$ The correlation of CRC-enriched bacteria with various pathways, such as lipopolysaccharide and energy biosynthesis, ${ }^{17}$ protein and mucin catabolism, and carbohydrate degradation, ${ }^{18}$ provides insights into their functional capacity in CRC. Moreover, compelling evidence suggests that microbial shifts are highly distinct across tumour stages. Two patterns of species elevation have been reported: the first consisted of a continuous increase from early stages onwards, while the other showed elevation only in early stages. The shifts in the microbiome and metabolome occurred at early stages may provide clues for the aetiology of CRC. ${ }^{19}$ An influx of bacterial species originating from the oral cavity has been suggested to play a role in CRC pathogenesis as the gut microbiome of patients with CRC exhibits higher species richness than that of control subjects, with the expansion of oral cavity-associated species rarely present in the healthy gut. ${ }^{20}$ In parallel, these large-scale, cross-cohort studies on faecal metagenomes not only establish a link between the gut microbiome and CRC but also identify microbiome signatures for CRC that can accurately predict disease across multiple datasets. Microbial CRC biomarkers
To cite: Cheng WY, Wu C-Y, Yu J. Gut

2020;69:1867-1876 
identified by cross-cohort studies potentially enhance noninvasive microbial diagnostic tests, distinguishing CRC cases from normal individuals.

Series of in vitro and in vivo studies further highlight the mechanisms used by the gut microbiota to promote tumourigenesis. Gavage of faecal samples from patients with CRC promotes colon carcinogenesis in germ-free and conventional mice. ${ }^{21}$ Additional mouse models demonstrate that depleting the microbiota suppresses lung cancer development induced by Kras mutation and p53 loss. ${ }^{11}$ Bacteria have been reported to promote inflammation and cancer cell proliferation via cytokine production. ${ }^{11}{ }^{22}$ Indeed, H.pylori and interleukin-22 (IL-22) induce matrix metalloproteinase-10 (MMP-10) in gastric epithelial cells via the extracellular signal-regulated kinase (ERK) pathway. MMP-10 not only induces inflammation through the production of chemokine ligand 16 (CXCL16) and recruitment of $\mathrm{CD}^{+} \mathrm{T}$ cells but also damages the gastric mucosa by inhibiting tight junction proteins. ${ }^{22}$ Besides animal models, gastric organoids show that the virulence factor CagA of $H$. pylori associates with c-Met receptor and cellular proliferation. ${ }^{23}$ Together, these studies illustrate the interaction of bacteria with different components of the tumour microenvironment to promote tumourigenesis.

Chronic inflammation plays an important role in carcinogenesis and colitis-susceptible IL-10-deficient mouse model identifies the gut microbiota as a target of inflammation that influences the progression of CRC. ${ }^{24}$ Colitis promotes tumourigenesis by altering the microbiota and inducing the expansion of bacteria with genotoxic capabilities. ${ }^{24}$ Enterotoxigenic Bacteroides fragilis (ETBF) has been reported to promote colon tumourigenesis by stimulating exaggerated immune responses via $\mathrm{T}$ helper 17 (Th17) cells in mouse CRC model. ${ }^{25}$ Gavaging mice that are prone to develop colon tumour with Peptostreptococcus anaerobius, another bacteria implicated in CRC, demonstrates that P. anaerobius not only interacts with toll-like receptor 2 (TLR2) and TLR4 on colon cells, but also promotes CRC via the modulation of various immune cell types, including myeloid-derived suppressor cells (MDSCs), tumour-associated macrophages (TAMs) and granulocytic tumour-associated neutrophils. ${ }^{26} 27$ Moreover, Fusobacterium nucleatum, one of the key drivers of $\mathrm{CRC}$, has been shown to induce inflammatory and oncogenic responses via its virulence factors including FadA, Fap2 and LPS. $^{28} 29$

In addition to its function in modulating inflammation, the microbiota influences the efficacy of cancer treatment. Preclinical data from a colon cancer mouse model identified Gammaproteobacteria as the key bacteria that metabolise gemcitabine, a chemotheraoeutic drug, into its inactive form via cytidine deaminase. ${ }^{30}$ Human pancreatic ductal adenocarcinoma (PDAC) samples also contained elevated level of Gammaproteobacteria compared with normal pancreas. Culturing the bacteria from fresh human PDAC tumours with human colon carcinoma cell lines rendered the cell lines fully resistant to gemcitabine. ${ }^{30}$ The detection of Gammaproteobacteria may explain the gemcitabine resistance in PDAC patients and targeting these bacteria may sensitise tumour to gemcitabine treatment. Collectively, these findings describe the interactions between the gut microbiota and the host, which influence the process of tumourigenesis as well as the efficacy of cancer treatment. Alexander et al proposed a 'TIMER' mechanistic framework to illustrate how bacteria influence chemotherapy and the host in terms of translocation, immunomodulation, metabolism, enzymatic degradation, reduced diversity and ecological variation. ${ }^{31} 32$ The modulation of cancer immunotherapies by the microbiota has also been reported. ${ }^{33-37}$ In the following sections, we will explore the mechanisms used by distinct bacterial species to modulate the efficacy of chemotherapeutic drugs or immune checkpoint inhibitors (ICIs). Next, we will describe the diverse approaches that enhance cancer treatment via altering the microbiota, and discuss the potential clinical application of such approaches.

\section{BACTERIA THAT MAY IMPROVE CANCER THERAPY EFFICACY}

Multiple studies demonstrate that the therapeutic efficacy was diminished in the absence of the gut microbiota, suggesting that, through different mechanisms, commensal microbes modulate the anticancer immune responses induced by the therapies. Cyclophosphamide (CTX), an approved chemotherapeutic drug, has been shown to alter the composition of intestinal microbiota in mice and promote the translocation of specific Gram-positive bacteria into secondary lymphoid organs, stimulating the production of 'pathogenic' Th17 cells, which share hallmarks of T helper 1 (Th1) cells and Th17 cells. ${ }^{37}$ Removal of the gut microbiota in germ-free mice or mice that have been treated with antibiotics leads to drug resistance to CTX. ${ }^{37}$ On the other hand, genetic models consisting of Escherichia coli and Caenorhabditis elegans were used to elucidate the complex interactions among the host, bacteria and fluoropyrimidines, antimetabolite drugs commonly used to treat cancer. ${ }^{32}$ Bacteria modulate the efficacy of fluoropyrimidines in C.elegans via bacterial vitamin $\mathrm{B}_{6}, \mathrm{~B}_{9}$ and ribonucleotide metabolism (figure 1). However, the role of bacteria and dietary nutrients in drug efficacy remains elusive due to the complex nature of human body influenced by intrinsic factors as well as environmental factors.

Bacteria modulate the efficacy of not only chemotherapeutic drugs but also immunotherapy. An early study on mice receiving antibiotic cocktail supports that altered microbiota impairs CpGoligonucleotide immunotherapy and platinum chemotherapy. With an intact microbiota, the immunotherapy suppresses tumour growth via tumour necrosis factor (TNF) production by myeloid cells and CD8 T cell response. Conversely, antibiotic treatment impairs TNF and cytokine production by immune cells including monocytes, macrophages and dendritic cells, and reduces tumour regression in mice receiving immunotherapy. These findings suggest that, through TLR4 activation, commensal microbes stimulate tumour-associated myeloid cells to produce inflammatory cytokines in response to immunotherapy and thereby improve patients' outcome. ${ }^{36}$ Deeper mechanism insights into the functions of commensal bacteria, as well as their interactions with the host, promote the design of microbial consortia that will improve the efficacy of cancer therapies.

\section{BACTERIA THAT MAY PROMOTE CANCER GROWTH AND PROGRESSION}

In contrast to the commensal microbes that can boost immune cells to fight cancer, subsets of pathogenic bacteria are associated with the development and progression of tumours. Using subcutaneous and liver metastases models of pancreatic cancer, colon cancer and melanoma, Sethi et al showed that antibiotics-mediated suppression of tumour growth involved adaptive immunity. This inhibitory effect was attenuated in Rag1-knockout mice that were deficient in mature T and B cells. Mechanistically, targeting the microbiota led to increased level of interferon-gamma (IFN- $\gamma$ )-producing $\mathrm{T}$ cells and decreased level of IL-17a- and IL-10-producing T cells. ${ }^{39}$ Targeting the pathogenic bacteria may restore immune functions and augment the efficacy of cancer therapies.

Fusobacterium nucleatum has been implicated in various types of cancer, including colorectal cancer, ${ }^{19}$ 40-43 oesophageal 


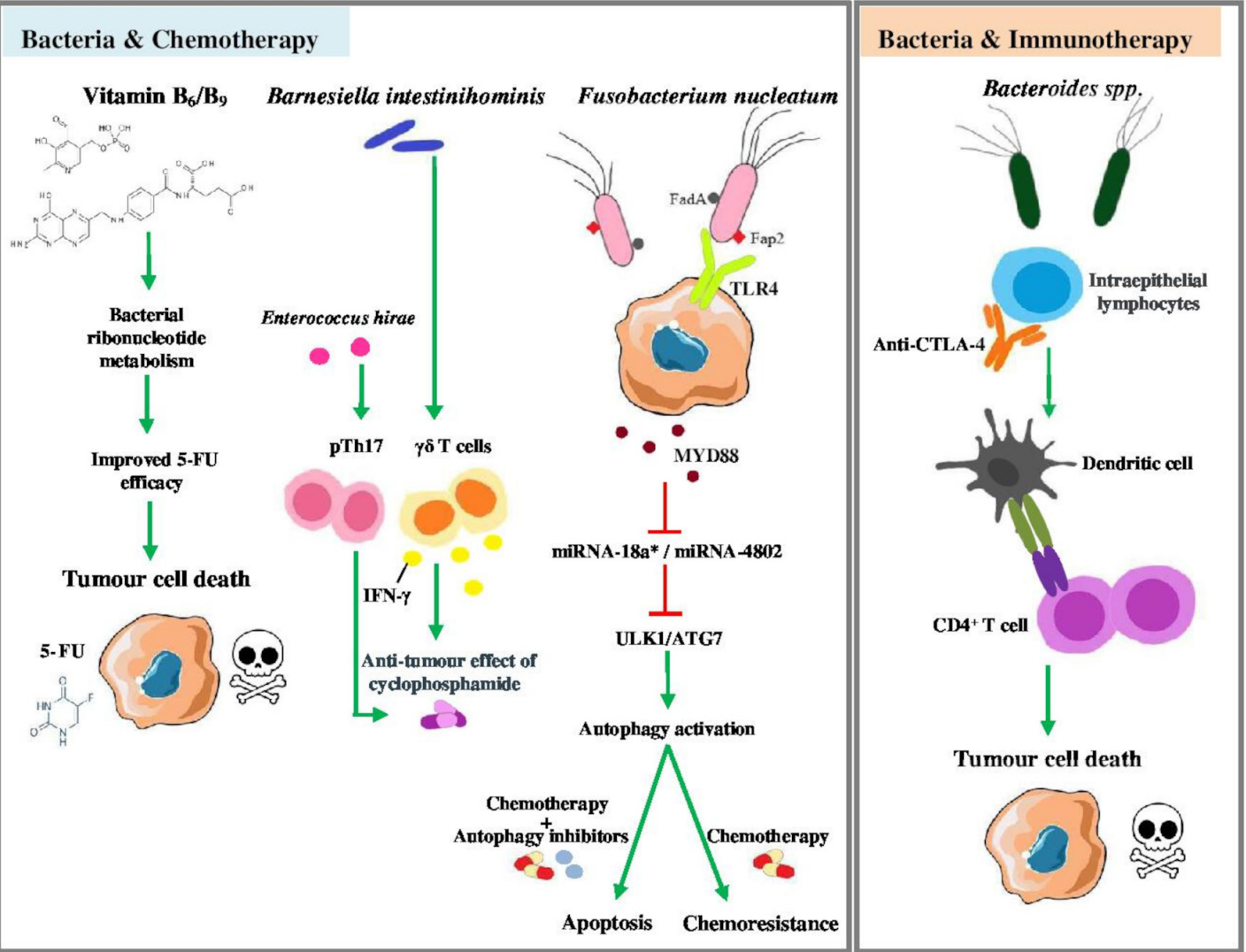

Figure 1 Mechanisms used by gut bacteria to modulate anticancer drug efficacy. Bacterial species influence the efficacy of chemo drugs and immune checkpoint inhibitors via diverse mechanisms. Bacteria modulate the efficacy of 5-FU via bacterial vitamin $B_{6}, B_{9}$ and ribonucleotide metabolism. Inhibiting bacterial deoxynucleotide metabolism promotes the efficacy of 5-FU. Barnesiella intestinihominis and Enterococcus hirae play a critical role in the antitumour effect of another chemo drug, cyclophosphamide. Removing these bacterial species results in drug resistance to cyclophosphamide. Mechanistically, E. hirae translocates from the small intestine to secondary lymphoid organs and stimulates the production of pTh17 cells, whereas B. intestinihominis accumulates in the colon and promotes the infiltration of IFN- $\gamma$-producing $\gamma \delta T$ cells in cancer lesions on treatment with cyclophosphamide. In addition, Fusobacterium nucleatum acts via TLR4 and MYD88, induces a selective loss of miR-18a* and miR4802 , activates autophagy and thereby promotes chemoresistance in patients. Furthermore, the antitumour effect of anti-CTLA-4 treatment depends on Bacteroides species. Bacteroides fragilis colonises the mucosal layer, induces T helper 1 immune responses in the lymph nodes and promotes the maturation of intratumorous dendritic cells, mediating the anticancer activity of the immune checkpoint inhibitor. 5-FU, 5-fluorouracil; IFN- $\gamma$, interferon-gamma; pTh17 cells, pathogenic T helper 17 cells.

cancer, ${ }^{44}$ gastric cancer, ${ }^{45}$ head and neck squamous cell carcinoma, ${ }^{46}$ and pancreatic cancer. ${ }^{47}$ Studies with cell lines and mouse models have demonstrated that FadA adhesin on F. nucleatum binds to epithelial cadherin (E-cadherin), induces $\beta$-catenin signalling and modulates inflammatory and oncogenic responses to promote tumourigenesis. Abolishing the binding of F. nucleatum to E-cadherin by synthetic peptide suppresses CRC cell growth. In concordance with the preclinical data, patients with CRC display an elevated level of fadA in their colon tissues compared with normal individuals. Further examination of patients' RNA revealed concomitant induction of Wnt7b and NFkB2 expression, suggesting a critical role of fadA in F. nucleatum-induced oncogenic and inflammatory responses. ${ }^{28}$ Another mechanism used by F.nucleatum to promote cancer growth is illustrated by studies that examined the functions of immune cells in the presence of F.nucleatum. These studies revealed that Fap 2 protein of F.nucleatum inhibited natural killer (NK) cell cytotoxicity via its interaction with T cell immunoglobulin and ITIM domain (TIGIT), regulated the localisation of the bacteria to colorectal tumours and induced cell death in human lymphocytes. ${ }^{48-50}$ Besides its pro-tumourigenic effects, F. nucleatum contributes to chemoresistance and it was enriched in CRC patients with recurrence post-chemotherapy. ${ }^{40}$ Mechanistically, F.nucleatum acts via TLR4 and MYD88, induces a selective loss of miR-18a* and miR-4802, activates autophagy and thereby promotes chemoresistance in patients with CRC. ${ }^{40}$ Targeting these F.nucleatum-associated pathways may ameliorate CRC patient outcomes (figure 1).

Similar to F.nucleatum, the enrichment of P.anaerobius has been associated with CRC. P.anaerobius adheres to cancer cells but not normal colonic epithelial cells via its surface protein, putative cell wall binding repeat 2 (PCWBR2), which interacts with $\alpha_{2} / \beta_{1}$ integrin expressed on colonic cells, and subsequently activates PI3K-Akt pathway, promoting cell proliferation as well as inflammation via nuclear factor kappa-light-chain-enhancer of activated B cells (NF- $\kappa \mathrm{B}$ ) cascade. With the reported functions of $P$.anaerobius in promoting cell proliferation and in triggering a proinflammatory tumour microenvironment via the recruitment of tumour-infiltrating MDSCs and TAMs, ${ }^{27}$ efforts should be directed to examining the effect of this bacterial species on chemotherapies and cancer immunotherapies.

Furthermore, radiotherapy exerts potent immune modulatory effect via tumour-associated antigen cross-presentation to cytolytic $\mathrm{CD}^{+} \mathrm{T}$ cells and IFN- $\gamma .{ }^{51}$ A study using melanoma 
and lung cancer models revealed that vancomycin, an antibiotic targeting Gram-positive bacteria, potentiated the radiotherapyinduced antitumour response in mice by increasing $\mathrm{CD} 8^{+} \mathrm{T}$ cell infiltration and IFN- $\gamma$ expression, whereas the supplementation of sodium butyrate, a key metabolite of Gram-positive bacteria, abolished the effect. ${ }^{51}$ The level of Gram-positive bacteria potentially influences patient's response to radiotherapy.

\section{MICROBIAL BIOMARKERS FOR THERAPY EFFICACY}

The antitumour immune response of CTX has been shown to be enhanced by several bacterial species, such as Lactobacillus johnsonii, Enterococcus hirae and Barnesiella intestinihominis, identified by mouse models. ${ }^{3752}$ Mechanistically, E. hirae translocates from the small intestine to secondary lymphoid organs and increases the intratumorous CD8/Treg ratio, whereas B.intestinihominis accumulates in the colon and promotes the infiltration of IFN- $\gamma$-producing $\gamma \delta$ T cells in cancer lesions. Oral gavage with E. hirae restores the antitumour effect of CTX, which is otherwise ablated by antibiotics treatment. Additional mouse model identified nucleotide-binding oligomerisation domaincontaining protein 2 as an inhibitor for the activity of E. hirae and B. intertinihominis. ${ }^{52}$

Similarly, the antitumour effect of anti-cytotoxic T-lymphocyteassociated protein 4 (CTLA-4) treatment depends on distinct Bacteroides species. Germ-free and antibiotics-treated mice do not respond to anti-CTLA-4 treatment; however, gavaging these mice with Bacteroides fragilis induces Th1 immune responses in the lymph nodes and promotes the maturation of intratumorous dendritic cells, restoring their response to CTLA blockade. Similar observation in patients reveals that the efficacy of CTLA-4 antibody is associated with $\mathrm{T}$ cell responses mediated by $B$. fragilis or Bacteroides thetaiotaomicron. ${ }^{35}$ In addition, Bifidobacterium breve, Bifidobacterium longum and several other species have been reported to augment dendritic cell function, and thereby induce $\mathrm{CD}^{+} \mathrm{T}$ cell priming and accumulation in the tumour microenvironment. ${ }^{33} 34$ The supplementation of these bacterial strains may enhance the therapeutic efficacy of ICIs in patients with cancer . Furthermore, bacteria have been shown to suppress melanoma growth by attenuating the unfolded protein response (UPR) signalling pathway and mediating antitumour immunity. Mice lacking the ubiquitin ligase RNF5 exhibit an altered gut microbiota that correlates with decreased expression of UPR but increased expression of inflammasome components. Transfer of 11 bacterial strains enriched in $R n f 5^{-/-}$mice promotes antitumour immunity and represses melanoma growth in germ-free wild-type mice. ${ }^{53}$ In accordance with the mouse data, patients that respond to immune checkpoint therapy exhibit significantly reduced level of UPR components. ${ }^{53}$ Modulation of selective bacterial species may enhance cancer immunotherapy and improve the outcomes of non-responders.

Complementing the preclinical mouse models, studies in patients highlight potential use of the gut microbiota in predicting treatment outcome. Analysing the faecal microbiota composition of metastatic melanoma (MM) patients receiving ipilimumab, an ICI targeting CTLA-4, revealed that patients with enriched level of Faecalibacterium and other Firmicutes were associated with not only longer survival but also increased occurrence of ipilimumab-induced colitis. ${ }^{54}$ Similar to CTLA-4, programmed cell death protein 1 (PD-1) is one of the inhibitory receptors which downregulate effector functions and suppress immune response. PD-1 blockade leads to significant tumour regression in a subset of patients with cancer. ${ }^{55-59}$ A metagenomic analysis of faecal samples from non-small cell lung cancer
(NSCLC) and renal cell carcinoma (RCC) patients receiving anti-PD-1 treatment indicated that non-responders had reduced levels of Akkermansia muciniphila, which had been shown to promote the recruitment of CCR9+CXCR3 + CD4+ T lymphocytes to the tumour microenvironment via IL-12 pathway. ${ }^{60}$ On the other hand, two studies in patients with melanoma validated the presence of commensal microbes that potentiated the antitumour effects of PD-1 blockade in responders. ${ }^{61}{ }^{62}$ Notably, responders display a higher alpha diversity and enriched levels of Ruminococcaceae and Faecalibacterium, which are associated with enhanced antigen presentation and $\mathrm{T}$ cell function in the tumour microenvironment. ${ }^{61}$ Taken together, a ratio of 'beneficial' bacteria to 'non-beneficial' bacteria is suggested to predict clinical response. ${ }^{62}$ Importantly, different cancer types, cancer treatments (chemotherapy vs immunotherapy), sample types (stool vs tissue) and approaches used to determine the microbial profile (16S vs metagenomic sequencing) may lead to biases and subsequently the identification of different species as beneficial bacteria. Faecalibacterium prausnitzii, for instance, plays a conflicting role in inflammation. F. prausnitzii has been associated with improved clinical response to immune checkpoint blockade, ${ }^{54}$ but it has also been shown to mitigate intestinal inflammation in the context of inflammatory bowel disease. ${ }^{64}$ With its ability to produce short-chain fatty acids (SCFAs), which induce Treg cells and IL-10, F.prausnitzii would not be expected to promote anticancer immune responses. However, a recent study demonstrated that SCFAs enhanced memory potential of activated $\mathrm{CD}^{+} \mathrm{T}$ cells, suggesting that F.prausnitzii may potentiate antitumour immune response by promoting $\mathrm{CD} 8^{+} \mathrm{T}$ cells' long-term survival as memory cells. ${ }^{65}$

Likewise, pathogenic bacteria can also serve as diagnostic or prognostic markers. Metagenomic data of patients with cancer indicated that F.nucleatum was enriched in CRC tissues compared with normal tissues. ${ }^{196667}$ Not only does the level of F. nucleatum increase with the degree of malignancy, ${ }^{40}$ but it also associates with metastasis. ${ }^{68} 69$ Further study of 309 subjects showed that complementing the faecal immunochemical test (FIT) with the detection of F. nucleatum improved diagnostic performance of FIT in colon cancer screening. ${ }^{70}$ Additional analysis of human CRC cohorts unveils other features of F.nucleatum-associated CRC, including rightsided colon location, $\mathrm{CpG}$ island methylation phenotype-high (CIMP-H), high level of microsatellite instability (MSI-H) and poor prognosis. ${ }^{71-73} \mathrm{CRC}$ with MSI-high status generally harbour numerous mutations and generate immunogenic peptides because of mismatch-repair deficiency. These alterations encourage antitumour immune response, leading to a more favourable prognosis and better response to immunotherapies. Considering these effects of MSI status, the Food and Drug Administration (FDA) approved the use of anti-PD1 antibodies, pembrolizumab and nivolumab, for CRC with high-MSI status. Interestingly, a study on 1041 patients with rectal and colon cancer demonstrated that the association of F.nucleatum with tumour-infiltrating lymphocytes (TIL) differed by tumour MSI status. The presence of F. nucleatum was negatively associated with TIL in MSI-high tumours, but positively associated with TIL in non-MSI-high tumours. The differential association of F.nucleatum with immune cells due to tumour MSI status suggests that F.nucleatum interacts with MSI status to mediate immune response within tumour. ${ }^{74}$ The F. nucleatuminduced changes in immune response influence cancer patients' treatment outcome and overall survival. Despite these observations in patients with cancer that strongly suggest the use of F. nucleatum as a prognostic marker, the relationships between 
F.nucleatum and methylation status of tumours, F.nucleatum and the right-sided colon location remain to be elucidated.

In addition to CRC, recent studies on liver cancer ${ }^{75}$ and pancreatic cancer ${ }^{1239}$ have elucidated the mechanisms used by bacteria to modulate immune profile and the efficacy of cancer treatment. Pushalkar et al demonstrated that human and mouse PDAC exhibited distinct bacterial composition compared with normal pancreas. Bacterial ablation with antibiotics reprogrammed the PDAC tumour microenvironment and enhanced the efficacy of ICI by reducing the level of MDSCs, inducing M1 macrophage differentiation and promoting $\mathrm{CD}^{+} \mathrm{T}$ cell activation. ${ }^{2}$ A recent study on liver cancer also delineated the mechanism used by bacteria to diminish antitumour immunity in the liver. Using multiple mouse models, Ma et al identified Clostridium species as important players in the regulation of bile acids, which in turn influenced the production of the chemokine CXCL16 by liver sinusoidal endothelial cells. CXCL16 recruits natural killer T (NKT) cells to the tumour, thereby inhibiting the growth of primary and metastatic liver cancer. ${ }^{75}$ In accordance with the preclinical data, bile acids were correlated with CXCL 16 expression in human liver sinusoidal endothelial cell line and in non-tumour liver tissues from patients with liver cancer. Targeting Clostridium species may restore antitumour immunity in the liver and suppress tumour growth. ${ }^{75}$

\section{MODULATION OF CANCER THERAPY-ASSOCIATED TOXICITIES BY THE MICROBIOTA}

GI surgery, including preoperative cleansing with oral and intravenous antibiotics, alters the microbiota and may lead to complications such as infection and anastomotic leakage. ${ }^{76} 77$ These complications may be ameliorated by modulating the microbiota as evidence suggests that specific bacteria, including Lactobacillus spp and A. muciniphila, regulate intestinal wound healing process via reactive oxygen species-dependent and formyl peptide receptors-dependent mechanisms. ${ }^{7678} 79$ A study using germ-free mice colonised with $B$. thetaiotaomicron also indicated the bacteria' function in regulating the expression of genes involved in nutrient absorption, mucosal barrier fortification and angiogenesis. ${ }^{80}$ In contrast to these beneficial bacteria that maintain intestinal function and epithelial integrity, pathogenic bacteria, such as Serratia marcescens and Pseudomonas aeruginosa, promote the development of anastomotic leakage. ${ }^{81} 82$ Therefore, selective inhibition of pathogens and simultaneous preservation of 'good' bacteria prior to or after surgery may minimise the risk of complications.

Similar to surgery, treatment with ICIs can result in adverse events including colitis. Metagenomic sequencing revealed that certain bacteria display altered profile in patients with cancer suffering from checkpoint-blockade-induced colitis. Of note, elevated level of Bacteroidetes and microbial genetic pathways involved in polyamine transport and B vitamin biosynthesis were shown to be associated with resistance to the development of colitis in patients with cancer receiving ipilimumab. The identified microbial biomarkers may predict patients' risk of developing ipilimumab-induced colitis. ${ }^{83}$ Supplementation of a cocktail of bacteria, including Bacteroidales and Burkholderiales, and faecal microbiota transplant (FMT) treatment have been shown to ameliorate ICI-induced colitis in antibiotic-treated mice and in cancer patients respectively. ${ }^{35}$ Although FMT reduces inflammation and ulceration with fewer side effects than corticosteroids, anti-TNF and anti-integrin agents, the extremely small sample size of the study and lack of mechanistic insight call for extensive validations. ${ }^{84}$
Analysis of the faecal samples of patients receiving radiotherapy suggested that patients with radiation enteropathy showed lower bacterial diversity but enrichment of specific microbial taxa, including Clostridium IV and Roseburia ${ }^{85}$ FMT has been shown to alleviate radiation-induced GI toxicity and increase the survival rate of irradiated mice by improving GI tract function and epithelial integrity. Notably, FMT thickens the mucus layer of irradiated mice by upregulating the expression of Muc2, Glut1 (Slc2a1), Pgk1, intestinal trefoil factor (TFF3/ITF1) and multidrug resistance protein 1 (MDR1). FMT also ameliorates radiation-induced bone marrow toxicity. Compared with FMT or bone marrow transplant alone, the combination treatment significantly increases the survival rate of irradiated mice, ${ }^{86}$ suggesting that FMT may serve as a radioprotector to improve prognosis of patients with cancer receiving radiotherapy.

In spite of its protective effect against cancer therapy-induced toxicities, FMT is associated with risks. Pathogens may be transmitted to the recipients via FMT. As such, robust microbiological screening is essential for abrogating the risk of transferring known pathogens, especially the ones carrying antimicrobial resistance. Nonetheless, it remains challenging to identify conditions for which FMT has true clinical potential. Limited data and knowledge on the role of FMT in cancer prevent the prediction of patient's response to FMT. Further analysis of the microbial profile of donors and recipients, as well as the associated mechanistic insights, is crucial for effective FMT treatment in patients. $^{87}$

\section{BACTERIA DEPLETION BY ANTIBIOTICS OR OTHER MEANS}

Given their contribution to tumourigenesis, carcinogenic bacteria have been depleted by various means to prevent cancer or delay tumour growth. Among these pathogens, $H$. pylori's role in gastric tumourigenesis is well-established. ${ }^{88} 89$ Eradication of $H$. pylori by a combination of amoxicillin and clarithromycin in patients with early gastric cancer is associated with lower rates of developing metachronous gastric cancer and improvements in the grade of glandular atrophy at the corpus. ${ }^{90}$ Conversely, an analysis of 125441 cancer cases and 490510 control cases indicated that antibiotic treatment positively correlated with cancer risk. ${ }^{91} 92$ Antibiotics have also been associated with reduced survival in patients with advanced renal cell carcinoma receiving anti-programmed cell death ligand-1 (PD-L1) monoclonal antibody (mAb) monotherapy, ${ }^{93}$ suggesting that the removal of commensals undermines the efficacy of ICIs.

The conventional use of antibiotics alters the commensal microbiota, leading to collateral damage to patients. Novel technologies, such as CRISPR-Cas 9 system delivered by phage, ${ }^{94} 95$ targeting specific bacteria at the microbiome-cancer interface are needed to minimise perturbation to the commensal microbiota, and to ensure effective cancer treatment. Notably, administration of irinotecan-loaded dextran nanoparticles covalently linked to azide-modified phages that target F. nucleatum in the tumours augments the effect of chemotherapy against CRC. The phage treatment is restricted to GI tumours, reducing potential toxicities associated with therapy. ${ }^{96}$ Ongoing clinical trials have proven the utility of nanoparticles in drug delivery with increasing number of nanomedicines being approved for various indications. $^{97}$

Nanotechnologies can be used to target tumour-associated bacteria or to release anticancer drugs in a controlled manner, causing fewer side effects in patients. ${ }^{9899}$ Given the implications of nanotechnologies for cancer prevention and treatment, efforts 
should be directed to evaluating toxicity, side effects and downstream mechanisms mediated by nanoparticles.

\section{BACTERIA COMPOSITIONAL MODULATION BY PROBIOTICS IN CANCER}

Numerous clinical trials have been conducted to investigate the effects of probiotics/prebiotics on cancer. Some trials ${ }^{10-103}$ reported an improved clinical outcome of patients receiving probiotics, whereas others ${ }^{104} 105$ failed to examine a significant effect induced by probiotics (online supplementary $S$ table 1 ).

A prospective intervention study on patients with CRC revealed that the administration of Lactobacillus acidophilus NCFM and Bifidobacterium lactis $\mathrm{Bl}-04$ altered patients' microbial profile. The probiotics increased the abundance of butyrate-producing bacteria, such as Faecalibacterium and Clostridiales spp, while decreasing the abundance of CRC-associated genera, including Fusobacterium and Peptostreptococcus. ${ }^{106}$ In addition to altering the microbial profile, probiotics have been reported to inhibit cancer progression in animal models. Using a diethylnitrosamine (DEN) model of rat hepatocarcinogenesis, Zhang et al demonstrated that the administration of the probiotic mixture VSL\#3 mitigated intestinal inflammation on DEN treatment, maintained the intestinal mucosa integrity and suppressed tumour growth. ${ }^{107}$ A subsequent study demonstrated that the probiotic mixture Prohep reduced the level of Th17 cells in the tumour, thereby inhibiting hepatocellular carcinoma (HCC) progression in a subcutaneous transplant mouse model. ${ }^{108}$ Clinical trials assessing the therapeutic potential of VSL\#3 in patients with cirrhosis $^{109}$ or non-alcoholic fatty liver disease ${ }^{110}$ suggested that probiotics improved the severity of the diseases that are strongly linked to the development of HCC. ${ }^{111}$

Recently, a study by Riehl et al delineated the mechanism of Lactobacillus rhamnosus GG (LGG) radioprotection. Tumourbearing mice were treated with phosphate buffered saline alone, irradiation alone, LGG alone or LGG followed by irradiation. The result demonstrated that LGG releases lipoteichoic acid (LTA), which binds to TLR2 on pericryptal macrophages and promotes chemokine CXCL12. CXCL12 in turn binds to CXCR4 on cyclo-oxygenase-2 expressing mesenchymal stem cells, driving their migration to the pericryptal region and producing prostaglandin E2 that protects epithelial stem cells from radiation-induced apoptosis. ${ }^{112}$ Yet, this mechanism remains to be validated in human.

In addition to radioprotection, probiotics may manipulate host inflammatory status, thereby influencing the efficacy of cancer therapy. ${ }^{113}$ Several clinical trials, however, deny the clinical benefits of probiotics in cancer treatment. ${ }^{114} 115$ Treating patients with head and neck cancer with a cocktail of Lactobacillus and Bifidobacterium strains after surgery did not improve the clinical outcome of patients. Patients receiving the probiotics and those receiving the placebo had similar rate of postoperative infection and displayed comparable level of inflammatory markers and diamine oxidase, an indicator of gut permeability. ${ }^{104}$ The conflicting results of clinical trials may be explained by interindividual variations in microbiome and host genome. Colonisation and function of probiotics are influenced by indigenous microbiota, gene-expression profile of the host and other exogenous factors. ${ }^{116-118}$ Due to these variations, it remains challenging to assess the clinical benefits of probiotics in patients with cancer. In addition, the majority of the clinical trials on probiotics shares limitations, such as small sample size, short duration of treatment and lack of follow-up to examine the long-term effect of probiotics on patients. Therefore, well-designed studies are critical for the evaluation of probiotic treatment in patients with cancer. Interestingly, a recent study investigated the effects of 11-strain probiotics or autologous faecal microbiome transplantation (aFMT) on post-antibiotic reconstitution of mouse and human microbiota. The result indicated that probiotics significantly delayed microbiome reconstitution, while aFMT induced a rapid and near-complete recovery within days of administration. On the basis of this finding, aFMT, instead of probiotics, may be used to reconstitute the microbiome in antibioticsperturbed patients. ${ }^{116}$

\section{ORGANOIDS PROVIDE DEEPER MECHANISTIC INSIGHTS INTO THE ROLE OF THE GUT MICROBIOTA IN CANCER}

Organoids cultured from pluripotent stem cells or tissues maintain many characteristics of their respective tissues, including histology and expression of markers. ${ }^{119}$ With these properties, organoids are adopted for disease modelling and drug screening. ${ }^{120}$ Organoids overcome drawbacks of current studies using cell lines or mouse models, including limited access to human samples and intrinsic differences between mouse models and human physiology. ${ }^{121}$ The addition of bacteria to organoid culture allows the study of host-bacteria interactions and offers a deeper mechanistic insight than two-dimensional culture.

A study confirmed the pro-tumourigenic effect of H.pylori via microinjection of the bacteria into human gastric organoids. The CagA protein of $\mathrm{H}$.pylori binds to the c-Met receptor of organoid epithelial cells, phosphorylates c-Met and promotes epithelial cell proliferation. ${ }^{23}$ Moreover, human monocytederived dendritic cells (DCs) were cocultured with gastric organoids and subsequently exposed to H.pylori. The result showed that DCs migrated to and interacted with gastric epithelial organoids and their migration was enhanced by $H$.pylori infection. ${ }^{122}$ This coculture system recapitulated what happened in vivo during a bacterial infection. DCs are recruited to the gastric mucosa on $\mathrm{H}$.pylori infection to promote phagocytosis of H.pylori. Furthermore, fusion of organoids and coculture of multiple organoids has recently been proposed to enhance the structural complexity and functional maturation of organoids. ${ }^{123}{ }^{124}$ Clinically, patient-derived organoids may be used for predicting tumour's sensitivity to various therapies.

\section{FUTURE PERSPECTIVES AND CONCLUSIONS}

With advances in sequencing technology and development of powerful computational tools, the research paradigm has shifted from association-based approach to mechanism-based approach. The mere presence or absences of certain bacterial species provides limited insight into the role of the gut microbiota in cancers. Indeed, unravelling causal links between bacteria and cancers, as well as the underlying mechanisms, has become the focus of intense research. Moreover, altered viromes and fungal microbiota have been implicated in CRC, ${ }^{125} 126$ suggesting that these microorganisms may interact with gut bacteria to modulate patients' response to cancer treatment. The integrated analysis of the gut microbiome and its interactions with the host, anticancer drugs and other exogenous factors ${ }^{127-129}$ is essential for improving the outcomes of cancer patients (online supplementary $S$ table 2).

Gnotobiotic animal models have been widely used to examine the colonisation of specific bacterial species in the gut and their biological functions in the context of cancer, and to provide the proof of principle needed to direct and interpret human studies. ${ }^{24}{ }^{130}$ However, differences in physiology of the intestinal tract, dietary patterns and genetics suggested that the mouse 


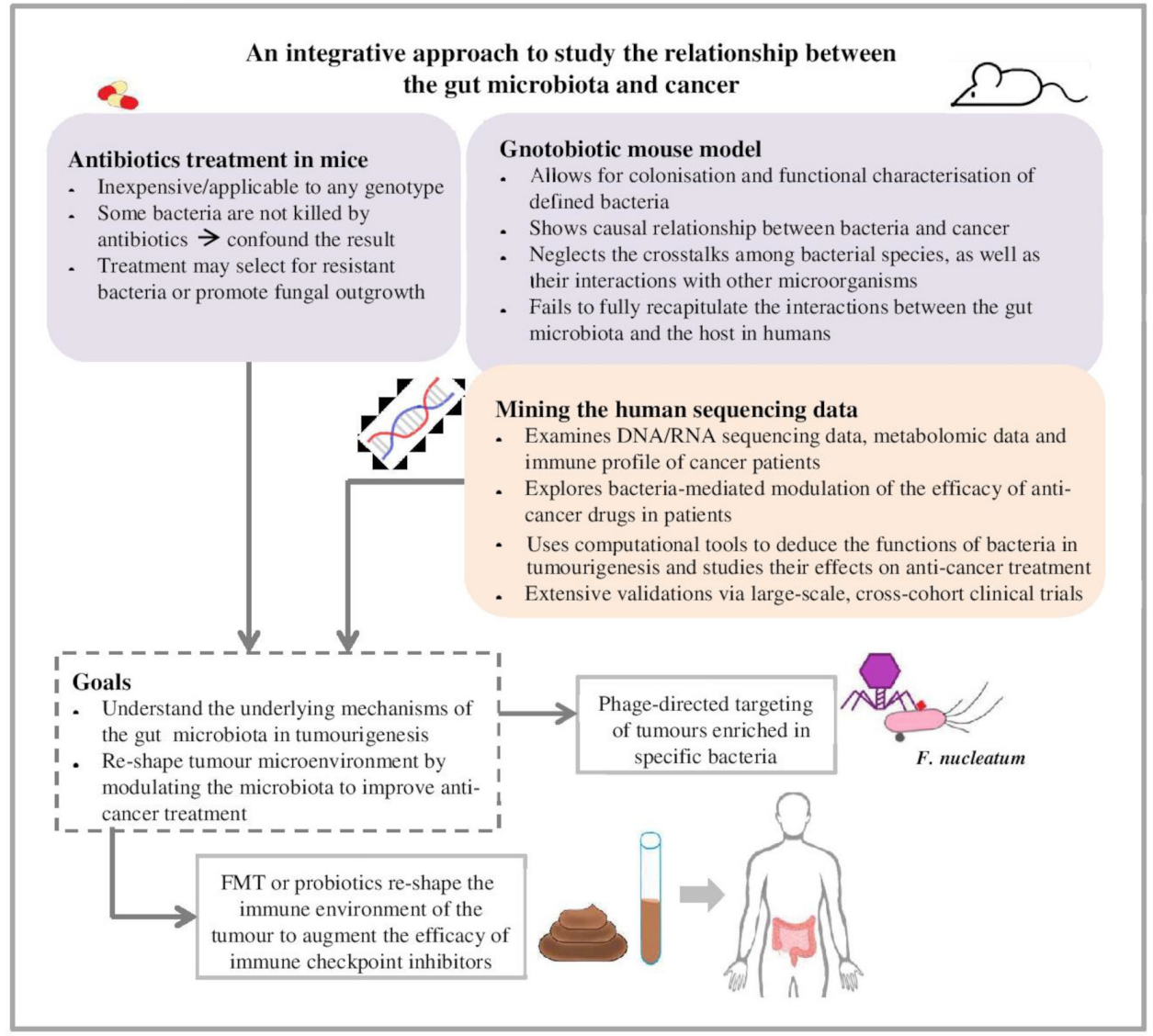

Figure 2 Preclinical mouse models and sequencing data of patients with cancer are valuable tools in microbiome research that contribute to the development of novel therapeutics for patients with cancer. Preclinical mouse models are valuable tools to dissect the mechanisms of defined bacteria in the absence of microbes (germ-free mice) or in the presence of limited number of microbes (antibiotic treatment). However, limitations, such as selection bias, prevent these preclinical models from fully recapitulating the interactions between the gut microbiota and cancer cells in humans. The antibiotic treatment may select for resistant bacteria or promote fungal outgrowth, confounding the result of the experiment. Other factors, including the housing environment, the diet and the genetic background of the mice, also influence the microbial communities residing in the mice and their interaction with tumour cells or anticancer drugs. To complement these preclinical models, cancer patients' sequencing data are examined in terms of the host genome, metabolome and immune profile before and after anticancer treatment. Together, the mouse models and meta-analysis of human sequencing data provide insights into the relationship between the gut microbiota and cancer treatment. Modulating the gut microbiota by selectively targeting cancer-associated bacteria with phages, by administering probiotics or by performing FMT, may reshape the tumour microenvironment and the host immune responses, thereby augmenting the efficacy of anticancer drugs and improving the outcome of cancer patients. FMT, faecal microbiota transplant.

microbiota differs significantly from the human microbiota. ${ }^{131} 132$ Reproducibility of mouse microbiota studies and their conclusions are to be interpreted with caution, and the findings are to be validated extensively by large-scale, multicentred clinical trials. Collaborative efforts to elucidate the role of the gut microbiota in shaping responses to cancer therapy are evident in several ongoing trials that evaluate the effect of FMT in patients with cancer who are impervious to ICIs. The teams conducting these trials propose that non-responders lack beneficial bacteria that are critical for the antitumour effect of immunotherapy, and that transplanting these bacteria from responding patients to non-responders restore their response to ICIs (NCT04130763, NCT04116775, NCT03353402, NCT03341143). These trials, along with further validations, will determine if selective modulation of gut microbiota, either by FMT, probiotic treatment or other means, enables patients with cancer to overcome resistance to immune checkpoint therapy (figure 2).

Accumulating evidence has underscored the critical role of the gut microbiota in cancer therapies. Some bacterial species are essential for the antitumour effect of cancer treatment, whereas other species attenuate the efficacy of cancer drugs via diverse mechanisms. Given the relationships between host, gut microbiota and cancer treatments, research efforts have been directed to investigate the mechanisms used by bacteria to modulate patients' response to treatment and their clinical outcomes. In this review, we explored the dynamic interactions between the host, the gut microbiota and various cancer treatments, including chemotherapies and immunotherapies. Regardless of the recent advances in the field of microbiome research, limitations of current research hamper the clinical applications of bacterial therapies. Of note, strong associations between distinct bacterial species and cancers have been established, but the underlying mechanisms remain elusive and need extensive validation via preclinical models and clinical trials. Nonetheless, recent findings supported the potential of microbial markers or enterotypes $^{133}$ in cancer diagnosis and prognosis, the potential of phage therapies in targeted delivery of cancer drugs, and the potential of FMT or probiotics in remodelling the tumour microenvironment or in potentiating antitumour immunity. The role of the microbiota in cancer is context-dependent. Bacteria 
interact with a plethora of host, as well as exogenous factors in patients with cancer and influence tumourigenesis via diverse mechanisms. Overall, a more comprehensive understanding of the functions of distinct bacteria in host physiology and in cancer treatment is critical to the development of personalised medicine that enhances patients' response to cancer therapies by modulating the gut microbial composition and function.

Contributors WYC researched data for the article, contributed substantially to discussion of content, designed the figures and wrote the article. C-YW reviewed/ edited the manuscript. JY designed the content and rewrite the manuscript.

Funding The authors have not declared a specific grant for this research from any funding agency in the public, commercial or not-for-profit sectors.

Competing interests None declared.

Patient consent for publication Not required.

Provenance and peer review Not commissioned; externally peer reviewed.

Open access This is an open access article distributed in accordance with the Creative Commons Attribution Non Commercial (CC BY-NC 4.0) license, which permits others to distribute, remix, adapt, build upon this work non-commercially, and license their derivative works on different terms, provided the original work is properly cited, appropriate credit is given, any changes made indicated, and the use is non-commercial. See: http://creativecommons.org/licenses/by-nc/4.0/.

ORCID iD

Jun Yu http://orcid.org/0000-0001-5008-2153

\section{REFERENCES}

1 Riquelme $\mathrm{E}$, Zhang $\mathrm{Y}$, Zhang $\mathrm{L}$, et al. Tumor microbiome diversity and composition influence pancreatic cancer outcomes. Cell 2019;178:795-806

2 Pushalkar S, Hundeyin M, Daley D, et al. The pancreatic cancer microbiome promotes oncogenesis by induction of innate and adaptive immune suppression. Cancer Discov 2018;8:403-16.

3 Virtue AT, McCright SJ, Wright JM, et al. The gut microbiota regulates white adipose tissue inflammation and obesity via a family of microRNAs. Sci Trans/ Med 2019;11:eaav1892.

4 Foley KP, Zlitni S, Denou E, et al. Long term but not short term exposure to obesity related microbiota promotes host insulin resistance. Nat Commun 2018:9:4681.

5 Sun L, Ma L, Ma Y, et al. Insights into the role of gut microbiota in obesity: pathogenesis, mechanisms, and therapeutic perspectives. Protein Cell 2018;9:397-403.

6 Koh A, Molinaro A, Ståhlman M, et al. Microbially produced imidazole propionate impairs insulin signaling through mTORC1. Cell 2018;175:947-61.

7 Brown K, Godovannyi A, Ma C, et al. Prolonged antibiotic treatment induces a diabetogenic intestinal microbiome that accelerates diabetes in NOD mice. Isme $J$ 2016;10:321-32.

8 Livanos AE, Greiner TU, Vangay P, et al. Antibiotic-Mediated gut microbiome perturbation accelerates development of type 1 diabetes in mice. Nat Microbiol 2016;1:16140

9 Maini Rekdal V, Bess EN, Bisanz JE, et al. Discovery and inhibition of an interspecies gut bacterial pathway for levodopa metabolism. Science 2019;364:eaau6323.

10 Erny $D$, Hrabě de Angelis AL, Jaitin D, et al. Host microbiota constantly control maturation and function of microglia in the CNS. Nat Neurosci 2015:18:965-77.

11 Jin C, Lagoudas GK, Zhao C, et al. Commensal microbiota promote lung cancer development via $\gamma \delta$ T cells. Cell 2019;176:e16:998-1013.

12 Tilg $H$, Adolph TE, Gerner RR, et al. The intestinal microbiota in colorectal cancer. Cancer Cell 2018:33:954-64.

13 Zitvogel L, Daillère R, Roberti MP, et al. Anticancer effects of the microbiome and its products. Nat Rev Microbiol 2017:15:465-78.

14 Garrett WS. Cancer and the microbiota. Science 2015;348:80-6.

15 Polk DB, Peek RM. Helicobacter pylori: gastric cancer and beyond. Nat Rev Cancer 2010:10:403-14

16 Wong BC-Y, Lam SK, Wong WM, et al. Helicobacter pylori eradication to prevent gastric cancer in a high-risk region of China: a randomized controlled trial. JAMA 2004;291:187-94.

17 Dai Z, Coker 00, Nakatsu G, et al. Multi-Cohort analysis of colorectal cancer metagenome identified altered bacteria across populations and universal bacteria markers. Microbiome 2018:6:70.

18 Wirbel J, Pyl PT, Kartal E, et al. Meta-Analysis of fecal metagenomes reveals global microbial signatures that are specific for colorectal cancer. Nat Med 2019;25:679-89.

19 Yachida S, Mizutani S, Shiroma H, et al. Metagenomic and metabolomic analyses reveal distinct stage-specific phenotypes of the gut microbiota in colorectal cancer. Nat Med 2019;25:968-76
20 Thomas AM, Manghi P, Asnicar F, et al. Metagenomic analysis of colorectal cancer datasets identifies cross-cohort microbial diagnostic signatures and a link with choline degradation. Nat Med 2019;25:667-78

21 Wong SH, Zhao L, Zhang $X$, et al. Gavage of fecal samples from patients with colorectal cancer promotes intestinal carcinogenesis in germ-free and conventional mice. Gastroenterology 2017;153:1621-33.

22 Lv Y-P, Cheng P, Zhang J-Y, et al. Helicobacter pylori-induced matrix metallopeptidase-10 promotes gastric bacterial colonization and gastritis. Sci Adv 2019:5:eaau6547.

23 McCracken KW, Catá EM, Crawford CM, et al. Modelling human development and disease in pluripotent stem-cell-derived gastric organoids. Nature 2014;516:400-4.

24 Arthur JC, Perez-Chanona E, Mühlbauer M, et al. Intestinal inflammation targets cancer-inducing activity of the microbiota. Science 2012:338:120-3.

25 Wu S, Rhee K-J, Albesiano E, et al. A human colonic commensal promotes colon tumorigenesis via activation of T helper type $17 \mathrm{~T}$ cell responses. Nat Med 2009;15:1016-22.

26 Tsoi $\mathrm{H}$, Chu ESH, Zhang $\mathrm{X}$, et al. Peptostreptococcus anaerobius induces intracellula cholesterol biosynthesis in colon cells to induce proliferation and causes dysplasia in mice. Gastroenterology 2017:152:1419-33.

27 Long X, Wong CC, Tong L, et al. Peptostreptococcus anaerobius promotes colorectal carcinogenesis and modulates tumour immunity. Nat Microbiol 2019;4:2319-30.

28 Rubinstein MR, Wang $X$, Liu W, et al. Fusobacterium nucleatum promotes colorectal carcinogenesis by modulating $E$-cadherin/ $\beta$-catenin signaling via its FadA adhesin. Cell Host Microbe 2013;14:195-206.

29 Gholizadeh P, Eslami H, Kafil HS. Carcinogenesis mechanisms of Fusobacterium nucleatum. Biomed Pharmacother 2017;89:918-25.

30 Geller LT, Barzily-Rokni M, Danino T, et al. Potential role of intratumor bacteria in mediating tumor resistance to the chemotherapeutic drug gemcitabine. Science 2017:357:1156-60.

31 Alexander JL, Wilson ID, Teare J, et al. Gut microbiota modulation of chemotherapy efficacy and toxicity. Nat Rev Gastroenterol Hepatol 2017:14:356-65.

32 Scott TA, Quintaneiro LM, Norvaisas P, et al. Host-Microbe Co-metabolism Dictates Cancer Drug Efficacy in C. elegans. Cell 2017:169:e18:442-56.

33 Tanoue T, Morita S, Plichta DR, et al. A defined commensal Consortium elicits CD8 T cells and anti-cancer immunity. Nature 2019:565:600-5.

34 Sivan A, Corrales L, Hubert N, et al. Commensal Bifidobacterium promotes antitumo immunity and facilitates anti-PD-L1 efficacy. Science 2015:350:1084-9.

35 Vétizou M, Pitt JM, Daillère R, et al. Anticancer immunotherapy by CTLA-4 blockade relies on the gut microbiota. Science 2015:350:1079-84.

36 lida N, Dzutsev A, Stewart CA, et al. Commensal bacteria control cancer response to therapy by modulating the tumor microenvironment. Science 2013:342:967-70.

37 Viaud S, Saccheri F, Mignot G, et al. The intestinal microbiota modulates the anticancer immune effects of cyclophosphamide. Science 2013;342:971-6.

38 Viaud S, Flament C, Zoubir M, et al. Cyclophosphamide induces differentiation of Th17 cells in cancer patients. Cancer Res 2011;71:661-5.

39 Sethi V, Kurtom S, Tarique M, et al. Gut microbiota promotes tumor growth in mice by modulating immune response. Gastroenterology 2018;155:33-7.

40 Yu T, Guo F, Yu Y, et al. Fusobacterium nucleatum promotes chemoresistance to colorectal cancer by modulating autophagy. Cell 2017;170:e16:548-63.

41 Fukugaiti $\mathrm{MH}$, Ignacio A, Fernandes MR, et al. High occurrence of Fusobacterium nucleatum and Clostridium difficile in the intestinal microbiota of colorectal carcinoma patients. Braz J Microbiol 2015;46:1135-40.

42 Castellarin M, Warren RL, Freeman JD, et al. Fusobacterium nucleatum infection is prevalent in human colorectal carcinoma. Genome Res 2012;22:299-306.

43 Kostic AD, Gevers D, Pedamallu CS, et al. Genomic analysis identifies association of Fusobacterium with colorectal carcinoma. Genome Res 2012;22:292-8.

44 Yamamura K, Baba Y, Nakagawa S, et al. Human microbiome Fusobacterium nucleatum in esophageal cancer tissue is associated with prognosis. Clin Cancer Res 2016:22:5574-81.

45 Hsieh Y-Y, Tung S-Y, Pan H-Y, et al. Increased abundance of Clostridium and Fusobacterium in gastric microbiota of patients with gastric cancer in Taiwan. Sci Rep 2018;8:158.

46 Shin JM, Luo T, Kamarajan P, et al. Microbial communities associated with primary and metastatic head and neck squamous cell carcinoma - a high Fusobacterial and low Streptococcal signature. Sci Rep 2017;7:9934

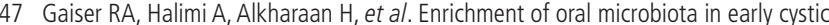
precursors to invasive pancreatic cancer. Gut 2019;68:2186-94.

48 Gur C, Ibrahim Y, Isaacson B, et al. Binding of the Fap2 protein of Fusobacterium nucleatum to human inhibitory receptor TIGIT protects tumors from immune cell attack. Immunity 2015:42:344-55.

49 Abed J, Emgård JEM, Zamir G, et al. Fap2 mediates Fusobacterium nucleatum colorectal adenocarcinoma enrichment by binding to tumor-expressed Gal-GalNAc Cell Host Microbe 2016:20:215-25.

50 Kaplan CW, Ma X, Paranjpe A, et al. Fusobacterium nucleatum outer membrane proteins Fap2 and RadD induce cell death in human lymphocytes. Infect Immun 2010;78:4774-8 
51 Uribe-Herranz M, Rafail S, Beghi S, et al. Gut microbiota modulate dendritic cell antigen presentation and radiotherapy-induced antitumor immune response. J Clin Invest 2020;130:466-79.

52 Daillère R, Vétizou M, Waldschmitt N, et al. Enterococcus hirae and Barnesiella intestinihominis facilitate cyclophosphamide-induced therapeutic immunomodulatory effects. Immunity 2016;45:931-43.

53 Li Y, Tinoco R, Elmén L, et al. Gut microbiota dependent anti-tumor immunity restricts melanoma growth in Rnf5 ${ }^{-/}$mice. Nat Commun 2019;10:1492.

54 Chaput N, Lepage P, Coutzac C, et al. Baseline gut microbiota predicts clinical response and colitis in metastatic melanoma patients treated with ipilimumab. Ann Oncol 2017;28:1368-79.

55 Ascierto PA, Long GV, Robert C, et al. Survival outcomes in patients with previously untreated BRAF wild-type advanced melanoma treated with nivolumab therapy: three-year follow-up of a randomized phase 3 trial. JAMA Oncol 2019:5:187-94.

56 Vokes EE, Ready N, Felip E, et al. Nivolumab versus docetaxel in previously treated advanced non-small-cell lung cancer (CheckMate 017 and CheckMate 057): 3-year update and outcomes in patients with liver metastases. Ann Oncol 2018;29:959-65.

57 Escudier B, Motzer RJ, Sharma P, et al. Treatment beyond progression in patients with advanced renal cell carcinoma treated with nivolumab in CheckMate 025. Eur Urol 2017;72:368-76.

58 Weber JS, D'Angelo SP, Minor D, et al. Nivolumab versus chemotherapy in patients with advanced melanoma who progressed after anti-CTLA-4 treatment (CheckMate 037): a randomised, controlled, open-label, phase 3 trial. Lancet Oncol 2015;16:375-84.

59 Topalian SL, Sznol M, McDermott DF, et al. Survival, durable tumor remission, and long-term safety in patients with advanced melanoma receiving nivolumab. J Clin Oncol 2014;32:1020-30.

60 Routy B, Le Chatelier E, Derosa L, et al. Gut microbiome influences efficacy of PD-1based immunotherapy against epithelial tumors. Science 2018;359:91-7.

61 Gopalakrishnan V, Spencer CN, Nezi L, et al. Gut microbiome modulates response to anti-PD-1 immunotherapy in melanoma patients. Science 2018;359:97-103.

62 Matson V, Fessler J, Bao R, et al. The commensal microbiome is associated with antiPD-1 efficacy in metastatic melanoma patients. Science 2018;359:104-8.

63 Frankel AE, Coughlin LA, Kim J, et al. Metagenomic shotgun sequencing and unbiased metabolomic profiling identify specific human gut microbiota and metabolites associated with immune checkpoint therapy efficacy in melanoma patients. Neoplasia 2017; 19:848-55.

64 Quévrain E, Maubert MA, Michon C, et al. Identification of an anti-inflammatory protein from Faecalibacterium prausnitzii, a commensal bacterium deficient in Crohn's disease. Gut 2016:65:415-25.

65 Bachem A, Makhlouf C, Binger KJ, et al. Microbiota-Derived Short-Chain Fatty Acids Promote the Memory Potential of Antigen-Activated CD8 ${ }^{+} \mathrm{T}$ Cells. Immunity 2019:51:285-97.

66 Lu Y, Chen J, Zheng J, et al. Mucosal adherent bacterial dysbiosis in patients with colorectal adenomas. Sci Rep 2016;6:26337.

67 Nakatsu G, Li X, Zhou H, et al. Gut mucosal microbiome across stages of colorectal carcinogenesis. Nat Commun 2015:6:8727.

68 Bullman S, Pedamallu CS, Sicinska E, et al. Analysis of Fusobacterium persistence and antibiotic response in colorectal cancer. Science 2017:358:1443-8.

69 Yan X, Liu L, Li H, et al. Clinical significance of Fusobacterium nucleatum, epithelialmesenchymal transition, and cancer stem cell markers in stage III/IV colorectal cancer patients. Onco Targets Ther 2017;10:5031-46.

70 Wong SH, Kwong TNY, Chow T-C, et al. Quantitation of faecal Fusobacterium improves faecal immunochemical test in detecting advanced colorectal neoplasia. Gut 2017:66:1441-8

71 Koi M, Okita Y, Carethers JM. Fusobacterium nucleatum Infection in Colorectal Cancer: Linking Inflammation, DNA Mismatch Repair and Genetic and Epigenetic Alterations. J Anus Rectum Colon 2018:2:37-46.

72 Mima K, Nishihara R, Qian ZR, et al. Fusobacterium nucleatum in colorectal carcinoma tissue and patient prognosis. Gut 2016;65:1973-80.

73 Tahara T, Yamamoto E, Suzuki H, et al. Fusobacterium in colonic flora and molecular features of colorectal carcinoma. Cancer Res 2014;74:1311-8.

74 Hamada T, Zhang X, Mima K, et al. Fusobacterium nucleatum in Colorectal Cancer Relates to Immune Response Differentially by Tumor Microsatellite Instability Status. Cancer Immunol Res 2018:6:1327-36.

$75 \mathrm{Ma} \mathrm{C}$, Han M, Heinrich B, et al. Gut microbiome-mediated bile acid metabolism regulates liver cancer via NKT cells. Science 2018;360:pii: eaan5931.

76 Bachmann R, Leonard D, Delzenne N, et al. Novel insight into the role of microbiota in colorectal surgery. Gut 2017;66:738-49.

77 Guyton K, Alverdy JC. The gut microbiota and gastrointestinal surgery. Nat Rev Gastroenterol Hepatol 2017;14:43-54.

78 Everard A, Belzer C, Geurts L, et al. Cross-Talk between Akkermansia muciniphila and intestinal epithelium controls diet-induced obesity. Proc Natl Acad Sci U SA 2013;110:9066-71.

79 Jones RM, Luo L, Ardita CS, et al. Symbiotic lactobacilli stimulate gut epithelial proliferation via Nox-mediated generation of reactive oxygen species. Embo J 2013;32:3017-28.
80 Hooper LV, Wong MH, Thelin A, et al. Molecular analysis of commensal hostmicrobial relationships in the intestine. Science 2001:291:881-4.

81 Hyoju SK, Klabbers RE, Aaron M, et al. Oral polyphosphate suppresses bacterial collagenase production and prevents anastomotic leak due to Serratia marcescens and Pseudomonas aeruginosa. Ann Surg 2018;267:1112-8.

82 van Praagh JB, de Goffau MC, Bakker IS, et al. Mucus microbiome of anastomotic tissue during surgery has predictive value for colorectal anastomotic leakage. Ann Surg 2019:269:911-6.

83 Dubin K, Callahan MK, Ren B, et al. Intestinal microbiome analyses identify melanoma patients at risk for checkpoint-blockade-induced colitis. Nat Commun 2016:7:10391.

84 Wang Y, Wiesnoski DH, Helmink BA, et al. Fecal microbiota transplantation for refractory immune checkpoint inhibitor-associated colitis. Nat Med 2018;24:1804-8.

85 Reis Ferreira M, Andreyev HJN, Mohammed K, et al. Microbiota- and radiotherapyinduced gastrointestinal side-effects (MARs) study: a large pilot study of the microbiome in acute and late-radiation enteropathy. Clin Cancer Res 2019;25:6487-500.

86 Cui M, Xiao H, Li Y, et al. Faecal microbiota transplantation protects against radiation-induced toxicity. EMBO Mol Med 2017:9:448-61.

87 Giles EM, D'Adamo GL, Forster SC. The future of faecal transplants. Nat Rev Microbiol 2019;17:719.

88 Romano M, Ricci V, Zarrilli R. Mechanisms of disease: Helicobacter pylorirelated gastric carcinogenesis--implications for chemoprevention. Nat Clin Pract Gastroenterol Hepatol 2006:3:622-32.

89 Peek RM, Blaser MJ. Helicobacter pylori and gastrointestinal tract adenocarcinomas. Nat Rev Cancer 2002:2:28-37.

90 Choi IJ, Kook M-C, Kim Y-I, et al. Helicobacter pylori therapy for the prevention of metachronous gastric cancer. N Engl J Med 2018:378:1085-95.

91 Boursi B, Mamtani R, Haynes K, et al. Recurrent antibiotic exposure may promote cancer formation--Another step in understanding the role of the human microbiota? Eur J Cancer 2015;51:2655-64.

92 Cao Y, Wu K, Mehta R, et al. Long-Term use of antibiotics and risk of colorectal adenoma. Gut 2018;67:672-8.

93 Derosa L, Hellmann MD, Spaziano M, et al. Negative association of antibiotics on clinical activity of immune checkpoint inhibitors in patients with advanced renal cell and non-small-cell lung cancer. Ann Oncol 2018;29:1437-44.

94 Bikard D, Euler CW, Jiang W, et al. Exploiting CRISPR-Cas nucleases to produce sequence-specific antimicrobials. Nat Biotechnol 2014;32:1146-50.

95 Citorik RJ, Mimee M, Lu TK. Sequence-Specific antimicrobials using efficiently delivered RNA-guided nucleases. Nat Biotechnol 2014;32:1141-5.

96 Zheng D-W, Dong X, Pan P, et al. Phage-guided modulation of the gut microbiota of mouse models of colorectal cancer augments their responses to chemotherapy. Nat Biomed Eng 2019;3:717-28.

97 Anselmo AC, Mitragotri S. Nanoparticles in the clinic: an update. Bioeng Trans/ Med 2019;4:e10143.

98 Song W, Anselmo AC, Huang L. Nanotechnology intervention of the microbiome for cancer therapy. Nat Nanotechnol 2019:14:1093-103.

99 Angsantikul P, Thamphiwatana S, Zhang Q, et al. Coating nanoparticles with gastric epithelial cell membrane for targeted antibiotic delivery against Helicobacter pylori infection. Adv Ther 2018;1:pii: 1800016

100 Flesch AT, Tonial ST, Contu PDEC, et al. Perioperative synbiotics administration decreases postoperative infections in patients with colorectal cancer: a randomized, double-blind clinical trial. Rev Col Bras Cir 2017;44:567-73.

101 Theodoropoulos GE, Memos NA, Peitsidou K, et al. Synbiotics and gastrointestinal function-related quality of life after elective colorectal cancer resection. Ann Gastroenterol 2016:29:56-62.

102 Mego M, Chovanec J, Vochyanova-Andrezalova l, et al. Prevention of irinotecan induced diarrhea by probiotics: a randomized double blind, placebo controlled pilot study. Complement Ther Med 2015;23:356-62.

103 Tian Y, Li M, Song W, et al. Effects of probiotics on chemotherapy in patients with lung cancer. Oncol Lett 2019;17:2836-48.

104 Lages PC, Generoso SV, Correia MITD. Postoperative symbiotic in patients with head and neck cancer: a double-blind randomised trial. Br J Nutr 2018;119:190-5.

105 Consoli MLD, da Silva RS, Nicoli JR, et al. Randomized clinical trial: impact of oral administration of Saccharomyces boulardii on gene expression of intestinal cytokines in patients undergoing colon resection. JPEN J Parenter Enteral Nutr 2016;40:1114-21.

106 Hibberd AA, Lyra A, Ouwehand AC, et al. Intestinal microbiota is altered in patients with colon cancer and modified by probiotic intervention. BMJ Open Gastroenterol 2017:4:e000145.

107 Zhang H-L, Yu L-X, Yang W, et al. Profound impact of gut homeostasis on chemicallyinduced pro-tumorigenic inflammation and hepatocarcinogenesis in rats. $J$ Hepatol 2012:57:803-12

108 Li J, Sung CYJ, Lee N, et al. Probiotics modulated gut microbiota suppresses hepatocellular carcinoma growth in mice. Proc Natl Acad Sci U SA 2016;113:E1306-15. 
109 Dhiman RK, Rana B, Agrawal S, et al. Probiotic VSL\#3 reduces liver disease severity and hospitalization in patients with cirrhosis: a randomized, controlled trial. Gastroenterology 2014;147:1327-37.

110 Alisi A, Bedogni G, Baviera G, et al. Randomised clinical trial: The beneficial effects of VSL\#3 in obese children with non-alcoholic steatohepatitis. Aliment Pharmacol Ther 2014;39:1276-85.

111 Michelotti GA, Machado MV, Diehl AM. Nafld, NASH and liver cancer. Nat Rev Gastroenterol Hepatol 2013;10:656-65.

112 Riehl TE, Alvarado D, Ee X, et al. Lactobacillus rhamnosus GG protects the intestinal epithelium from radiation injury through release of lipoteichoic acid, macrophage activation and the migration of mesenchymal stem cells. Gut 2019;68:1003-13.

113 Zaharuddin L, Mokhtar NM, Muhammad Nawawi KN, et al. A randomized doubleblind placebo-controlled trial of probiotics in post-surgical colorectal cancer. BMC Gastroenterol 2019;19:131.

114 Anderson ADG, McNaught CE, Jain PK, et al. Randomised clinical trial of synbiotic therapy in elective surgical patients. Gut 2004;53:241-5.

115 McNaught CE, Woodcock NP, MacFie J, et al. A prospective randomised study of the probiotic Lactobacillus plantarum $299 \mathrm{v}$ on indices of gut barrier function in elective surgical patients. Gut 2002;51:827-31.

116 Suez J, Zmora N, Zilberman-Schapira G, et al. Post-Antibiotic gut mucosal microbiome reconstitution is impaired by probiotics and improved by autologous FMT. Cell 2018;174:1406-23.

117 Zmora N, Zilberman-Schapira G, Suez J, et al. Personalized gut mucosal colonization resistance to empiric probiotics is associated with unique host and microbiome features. Cell 2018;174:e21:1388-405.

118 Morgan XC, Segata N, Huttenhower C. Biodiversity and functional genomics in the human microbiome. Trends Genet 2013;29:51-8.

119 Bartfeld S, Bayram T, van de Wetering M, et al. In vitro expansion of human gastric epithelial stem cells and their responses to bacterial infection. Gastroenterology 2015;148:126-36.
120 Broutier L, Mastrogiovanni G, Verstegen MM, et al. Human primary liver cancerderived organoid cultures for disease modeling and drug screening. Nat Med 2017;23:1424-35.

121 Prior N, Inacio P, Huch M. Liver organoids: from basic research to therapeutic applications. Gut 2019;68:2228-37.

122 Sebrell TA, Hashimi M, Sidar B, et al. A novel gastric spheroid co-culture model reveals chemokine-dependent recruitment of human dendritic cells to the gastric epithelium. Cell Mol Gastroenterol Hepatol 2019;8:157-71.

123 Jin Y, Kim J, Lee JS, et al. Vascularized liver organoids generated using induced hepatic tissue and dynamic liver-specific microenvironment as a drug testing platform. Adv Funct Mater 2018;28:1801954.

124 Koike $\mathrm{H}$, Iwasawa K, Ouchi R, et al. Modelling human hepato-biliary-pancreatic organogenesis from the foregut-midgut boundary. Nature 2019;574:112-6.

125 Coker 00, Nakatsu G, Dai RZ, et al. Enteric fungal microbiota dysbiosis and ecological alterations in colorectal cancer. Gut 2019;68:654-62.

126 Nakatsu G, Zhou H, Wu WKK, et al. Alterations in enteric virome are associated with colorectal cancer and survival outcomes. Gastroenterology 2018;155:529-41.

127 Zhernakova A, Kurilshikov A, Bonder MJ, et al. Population-Based metagenomics analysis reveals markers for gut microbiome composition and diversity. Science 2016;352:565-9.

128 Blaser MJ. Antibiotic use and its consequences for the normal microbiome. Science 2016;352:544-5.

129 David LA, Maurice CF, Carmody RN, et al. Diet rapidly and reproducibly alters the human gut microbiome. Nature 2014;505:559-63.

130 Lepage $\mathrm{P}$, Leclerc MC, Joossens $\mathrm{M}$, et al. A metagenomic insight into our gut's microbiome. Gut 2013;62:146-58.

131 Hugenholtz F, de Vos WM. Mouse models for human intestinal microbiota research: a critical evaluation. Cell Mol Life Sci 2018;75:149-60.

132 Xiao L, Feng Q, Liang S, et al. A catalog of the mouse gut metagenome. Nat Biotechnol 2015;33:1103-8.

133 Arumugam M, Raes J, Pelletier E, et al. Enterotypes of the human gut microbiome. Nature 2011;473:174-80. 